\title{
MODERATE DEVIATION AND LARGE DEVIATION FOR WEGMAN-DAVIES RECURSIVE DENSITY ESTIMATORS
}

\author{
BY \\ YU MIAO* (XINXIANG), QINGHUI GAO (XINXIANG), \\ JIANYONG MU (XINXIANG), AND CONGHUI DENG (XINXIANG)
}

\begin{abstract}
Let $\left\{X_{k}, k \geqslant 1\right\}$ be a sequence of independent identically distributed random variables with common probability density function $f$, and let $\hat{f}_{n}$ denote a Wegman-Davies recursive density estimator
\end{abstract}

$$
\hat{f}_{n}(x)=\frac{1}{n h_{n}^{1 / 2}} \sum_{j=1}^{n} \frac{1}{h_{j}^{1 / 2}} K\left(\frac{x-X_{j}}{h_{j}}\right)
$$

where $K$ is a kernel function and $h_{n}$ is a band sequence. In the present paper, the moderate deviation principle and the large deviation principle for the estimator $\hat{f}_{n}$ are established.

2020 Mathematics Subject Classification: Primary 62G07; Secondary $60 \mathrm{~F} 10$.

Key words and phrases: moderate deviation principle, large deviation principle, recursive kernel estimator.

\section{INTRODUCTION}

Given a sequence of independent identically distributed random variables $X_{1}, X_{2}, \ldots$ with common probability density function $f(x)$, how can one estimate $f(x)$ ? This problem has been of long-lasting interest among statisticians, and numerous interesting and fundamental results have been obtained.

1.1. Rosenblatt estimator. Rosenblatt [15] introduced the following kernel estimator of the density $f(x)$ :

$$
f_{n}^{*}(x)=\frac{1}{n h_{n}} \sum_{j=1}^{n} K\left(\frac{x-X_{j}}{h_{n}}\right),
$$

and Parzen [14] studied many important properties of these estimators, such as

* This work is supported by NSFC $(11471104,11971154)$.

(c) Probability and Mathematical Statistics, 2020 
consistency, asymptotic normality, uniform consistency, etc. Csörgö and Horváth [1] considered the central limit theorem for the $L_{p}$-norm of $f_{n}^{*}$. Lu [9] studied the kernel methods for density estimation of stationary samples under generalized conditions, which unify both the linear and $\alpha$-mixing processes and also apply to the non-linear and/or non- $\alpha$-mixing processes. Furthermore, under general, mild conditions, the estimators $f_{n}^{*}$ were shown to be asymptotically normal. Louani [8] established a large deviation limit theorem of Chernoff type for $f_{n}^{*}$ in the $L_{1}$-distance. Louani [7] and Gao [3] obtained the large deviation and moderate deviation principles for $f_{n}^{*}$ for pointwise and $L_{\infty}$ convergence. Mokkadem et al. [13] studied the large and moderate deviation principles for kernel estimators of the partial derivatives of $f$. Gao [4] proved the moderate deviation principle and the law of the iterated logarithm in $L_{1}\left(\mathbb{R}^{d}\right)$ for $f_{n}^{*}$.

1.2. Wolverton-Wagner estimator or Yamato estimator. It is well known that the Rosenblatt estimator $f_{n}^{*}$ is a non-recursive kernel density estimator. Wolverton and Wagner [17] defined a related estimator

$$
\tilde{f}_{n}(x)=\frac{1}{n} \sum_{j=1}^{n} \frac{1}{h_{j}} K\left(\frac{x-X_{j}}{h_{j}}\right),
$$

which was apparently independently introduced by Yamato [18]. The estimator $\tilde{f}_{n}(x)$ is useful in practice, because it can be calculated recursively,

$$
\tilde{f}_{n}(x)=\frac{n-1}{n} \tilde{f}_{n-1}(x)+\frac{1}{n h_{n}} K\left(\frac{x-X_{n}}{h_{n}}\right) .
$$

Masry and Györfi [11] obtained the sharp rates for almost sure convergence of $\tilde{f}_{n}$ when the process $\left\{X_{i}, i \geqslant 1\right\}$ is asymptotically uncorrelated. Liang and Baek [5], [6] discussed the point asymptotic normality and the Berry-Esseen bounds of $\tilde{f}_{n}$ for strictly stationary samples of negatively associated random variables. Mokkadem et al. [12] obtained the large and moderate deviation principles for the recursive kernel estimator of the probability density function $\tilde{f}_{n}$ and its partial derivatives in the multivariate case.

1.3. Wegman-Davies estimator. Wegman and Davies [16] introduced another related estimator $\hat{f}_{n}(x)$ by

$$
\hat{f}_{n}(x)=\frac{1}{n h_{n}^{1 / 2}} \sum_{j=1}^{n} \frac{1}{h_{j}^{1 / 2}} K\left(\frac{x-X_{j}}{h_{j}}\right),
$$

which can also be calculated recursively:

$$
\hat{f}_{n}(x)=\frac{n-1}{n}\left(\frac{h_{n-1}}{h_{n}}\right)^{1 / 2} \hat{f}_{n-1}(x)+\frac{1}{n h_{n}} K\left(\frac{x-X_{n}}{h_{n}}\right) .
$$


Masry and Györfi [11] established the sharp rates for almost sure convergence of $\hat{f}_{n}$ when the process $\left\{X_{i}, i \geqslant 1\right\}$ is asymptotically uncorrelated. Liang and Baek [6] discussed the Berry-Esseen bounds of $\hat{f}_{n}$ for strictly stationary samples of negatively associated random variables. Zhang and Liang [19] obtained the point asymptotic normality of $\hat{f}_{n}$ for such samples.

Motivated by that work, in the present paper we shall discuss the large deviation and the moderate deviation behaviour of the estimators $\hat{f}_{n}$.

\section{MODERATE DEVIATION PRINCIPLE}

We assume that $K$ is a Borel function satisfying

$$
\|K\|_{\infty}:=\sup _{y \in \mathbb{R}}|K(y)|<\infty, \quad \int_{\mathbb{R}}|K(y)| d y<\infty, \quad \lim _{y \rightarrow \pm \infty}|y K(y)|=0,
$$

and that $\left\{h_{n}\right\}$ is a sequence of positive real numbers satisfying

$$
h_{n} \downarrow 0, \quad n h_{n} \rightarrow \infty .
$$

Other assumptions about $K$ and $\left\{h_{n}\right\}$ will be made as needed. Firstly, we recall the following theorem of Wegman and Davies [16].

THEOREM 2.1 ([16, Theorem 1]). Let $K$ and $\left\{h_{n}\right\}$ satisfy 2.1) and (2.2).

(a) If $f$ is continuous at $x$, then

$$
n h_{n} \operatorname{Var}\left(\hat{f}_{n}(x)\right) \rightarrow f(x) \int_{\mathbb{R}} K^{2}(u) d u .
$$

(b) Let

$$
K^{*}(u)=\int_{\mathbb{R}} e^{-i u y} K(y) d y
$$

be the Fourier transform of $K$. Suppose that for some positive integer $\beta$,

$$
\lim _{u \rightarrow 0}\left[1-K^{*}(u)\right] /|u|^{\beta}=k_{\beta}
$$

is finite and the derivative $f^{(\beta)}(x)$ of order $\beta$ at $x$ exists. Suppose finally that

$$
n h_{n}^{\beta+1 / 2} \rightarrow \infty \quad \text { and } \quad \frac{1}{n h_{n}^{\beta+1 / 2}} \sum_{j=1}^{n} h_{j}^{\beta+1 / 2} \rightarrow \gamma_{\beta+1 / 2}
$$

Then

$$
\frac{\mathbb{E} \hat{f}_{n}(x)-f(x) n^{-1} h_{n}^{-1 / 2} \sum_{j=1}^{n} h_{j}^{1 / 2}}{h_{n}^{\beta}} \rightarrow \gamma_{\beta+1 / 2} \cdot k_{\beta} \cdot f^{(\beta)}(x) .
$$


REMARK 2.1. If there exists a value of $\beta$ such that $k_{\beta}$ is non-zero, it is called the characteristic exponent of $K^{*}(u)$, and $k_{\beta}$ is the characteristic coefficient.

THEOREM 2.2. Let $K$ and $\left\{h_{n}\right\}$ satisfy (2.1) and (2.2). Assume that a sequence $\left\{b_{n}\right\}$ of positive real numbers satisfies

$$
\lim _{n \rightarrow \infty} b_{n}=\infty, \quad \lim _{n \rightarrow \infty} \frac{b_{n}}{\sqrt{n h_{n}}}=0 .
$$

If $f$ is continuous at $x$, then for any $r>0$,

$$
\lim _{n \rightarrow \infty} \frac{1}{b_{n}^{2}} \log \mathbb{P}\left(\frac{\sqrt{n h_{n}}}{b_{n}}\left|\hat{f}_{n}(x)-\mathbb{E} \hat{f}_{n}(x)\right| \geqslant r\right)=-\frac{r^{2}}{2 \sigma^{2}(x)}
$$

where

$$
\sigma^{2}(x)=f(x) \int_{-\infty}^{\infty} K^{2}(u) d u .
$$

Proof. For any $r>0$, we will prove

$$
\frac{1}{b_{n}^{2}} \log \mathbb{P}\left(\frac{\sqrt{n h_{n}}}{b_{n}}\left(\hat{f}_{n}(x)-\mathbb{E} \hat{f}_{n}(x)\right) \geqslant r\right) \rightarrow-\frac{r^{2}}{2 \sigma^{2}(x)} ;
$$

the proof of

$$
\frac{1}{b_{n}^{2}} \log \mathbb{P}\left(\frac{\sqrt{n h_{n}}}{b_{n}}\left(\hat{f}_{n}(x)-\mathbb{E} \hat{f}_{n}(x)\right) \leqslant-r\right) \rightarrow-\frac{r^{2}}{2 \sigma^{2}(x)}
$$

is similar. For any $\lambda \in \mathbb{R}$, let

$$
\Lambda(\lambda):=\frac{\lambda^{2} \sigma^{2}(x)}{2} .
$$

The Fenchel-Legendre transform of $\Lambda(\cdot)$ is

$$
\Lambda^{*}(r):=\sup _{\lambda \in \mathbb{R}}\{\lambda r-\Lambda(\lambda)\}=\sup _{\lambda \in \mathbb{R}}\left\{\lambda r-\frac{\lambda^{2} \sigma^{2}(x)}{2}\right\}=\frac{r^{2}}{2 \sigma^{2}(x)}, \quad r \in \mathbb{R} .
$$

Let $Z_{n}:=\frac{\sqrt{n h_{n}}}{b_{n}}\left(\hat{f}_{n}(x)-\mathbb{E} \hat{f}_{n}(x)\right)$, and let $\Lambda_{n}(\cdot)$ be the logarithmic moment generating function of $Z_{n}$. By the Gärtner-Ellis theorem [2, Theorem 2.3.6], it is enough to show that for any $\lambda \in \mathbb{R}$,

$$
\Lambda(\lambda)=\lim _{n \rightarrow \infty} \frac{1}{b_{n}^{2}} \Lambda_{n}\left(\lambda b_{n}^{2} Z_{n}\right) .
$$

Define

$$
Y_{j}:=h_{j}^{-1 / 2}\left(K\left(\frac{x-X_{j}}{h_{j}}\right)-\mathbb{E} K\left(\frac{x-X_{j}}{h_{j}}\right)\right)
$$


Then we have

$$
\begin{aligned}
\frac{1}{b_{n}^{2}} \Lambda_{n}\left(\lambda b_{n}^{2} Z_{n}\right) & =\frac{1}{b_{n}^{2}} \log \mathbb{E} \exp \left(\lambda b_{n} \sqrt{n h_{n}}\left(\hat{f}_{n}(x)-\mathbb{E} \hat{f}_{n}(x)\right)\right) \\
& =\frac{1}{b_{n}^{2}} \sum_{j=1}^{n} \log \mathbb{E} \exp \left(\frac{\lambda b_{n}}{\sqrt{n}} h_{j}^{-1 / 2}\left(K\left(\frac{x-X_{j}}{h_{j}}\right)-\mathbb{E} K\left(\frac{x-X_{j}}{h_{j}}\right)\right)\right) \\
& =\frac{1}{b_{n}^{2}} \sum_{j=1}^{n} \log \mathbb{E} \exp \left(\frac{\lambda b_{n}}{\sqrt{n}} Y_{j}\right)
\end{aligned}
$$

and

$$
\begin{aligned}
\mid \frac{1}{b_{n}^{2}} \sum_{j=1}^{n} \log \mathbb{E} \exp ( & \left.\frac{\lambda b_{n}}{\sqrt{n}} Y_{j}\right)-\frac{\lambda^{2} \sigma^{2}(x)}{2} \mid \\
\leqslant & \frac{1}{b_{n}^{2}} \sum_{j=1}^{n}\left|\log \mathbb{E} \exp \left(\frac{\lambda b_{n}}{\sqrt{n}} Y_{j}\right)-\left[\mathbb{E} \exp \left(\frac{\lambda b_{n}}{\sqrt{n}} Y_{j}\right)-1\right]\right| \\
& +\frac{1}{b_{n}^{2}}\left|\sum_{j=1}^{n}\left[\left(\mathbb{E} \exp \left(\frac{\lambda b_{n}}{\sqrt{n}} Y_{j}\right)-1\right)-\frac{\lambda^{2} b_{n}^{2} \sigma^{2}(x)}{2 n}\right]\right| \\
= & : \triangle_{1}+\triangle_{2} .
\end{aligned}
$$

Now we need to control the terms $\triangle_{1}$ and $\triangle_{2}$. For $\triangle_{2}$ we can write

$$
\begin{aligned}
\triangle_{2}= & \frac{1}{b_{n}^{2}}\left|\sum_{j=1}^{n}\left[\left(\mathbb{E} \exp \left(\frac{\lambda b_{n}}{\sqrt{n}} Y_{j}\right)-1\right)-\frac{\lambda^{2} b_{n}^{2} \sigma^{2}(x)}{2 n}\right]\right| \\
\leqslant & \frac{1}{b_{n}^{2}} \sum_{j=1}^{n}\left|\mathbb{E} \exp \left(\frac{\lambda b_{n}}{\sqrt{n}} Y_{j}\right)-1-\frac{\lambda^{2} b_{n}^{2}}{2 n} \mathbb{E} Y_{j}^{2}\right| \\
& +\frac{1}{b_{n}^{2}}\left|\sum_{j=1}^{n} \frac{\lambda^{2} b_{n}^{2}}{2 n} \mathbb{E} Y_{j}^{2}-\frac{\lambda^{2} b_{n}^{2} \sigma^{2}(x)}{2 n}\right| \\
= & : \triangle_{21}+\triangle_{22} .
\end{aligned}
$$

For the term $\triangle_{21}$, by using the elementary inequality

$$
\left|e^{x}-1-x-\frac{x^{2}}{2}\right| \leqslant \frac{|x|^{3}}{6} e^{|x|} \quad \text { for all } x \in \mathbb{R},
$$

the condition (2.4) and the fact that $K(\cdot)$ is a bounded Borel function (which implies $\left.\left|Y_{i}\right| \leqslant 2 h_{i}^{-1 / 2}\|K\|_{\infty}\right)$, for any $n$ large enough we get

$$
\begin{aligned}
\triangle_{21} & =\frac{1}{b_{n}^{2}} \sum_{j=1}^{n}\left|\mathbb{E} \exp \left(\frac{\lambda b_{n}}{\sqrt{n}} Y_{j}\right)-1-\frac{\lambda^{2} b_{n}^{2}}{2 n} \mathbb{E} Y_{j}^{2}\right| \\
& \leqslant \frac{1}{b_{n}^{2}} \sum_{j=1}^{n} \frac{1}{6} \frac{\lambda^{3} b_{n}^{3}}{n^{3 / 2}} \mathbb{E}\left|Y_{j}^{3}\right| \exp \left(\frac{\lambda b_{n}}{\sqrt{n}}\left|Y_{j}\right|\right) \\
& \leqslant C_{0} \frac{b_{n} \lambda^{3}}{n^{1 / 2}} \frac{1}{n} \sum_{j=1}^{n} \mathbb{E}\left|Y_{j}\right|^{3} \leqslant C_{1} \frac{b_{n} \lambda^{3}}{n^{1 / 2} h_{n}^{1 / 2}} \frac{1}{n} \sum_{j=1}^{n} \mathbb{E} Y_{j}^{2}
\end{aligned}
$$


where $C_{0}, C_{1}$ are positive constants depending on the function $K(\cdot)$. So by using 2.4) again, and the fact (Theorem 2.1) that

$$
\begin{aligned}
\frac{1}{n} \sum_{j=1}^{n} \mathbb{E} Y_{j}^{2} & =\frac{1}{n} \sum_{j=1}^{n} h_{j}^{-1} \mathbb{E}\left(K\left(\frac{x-X_{j}}{h_{j}}\right)-\mathbb{E} K\left(\frac{x-X_{j}}{h_{j}}\right)\right)^{2} \\
& =n h_{n} \operatorname{Var}\left(\hat{f}_{n}(x)\right) \rightarrow \sigma^{2}(x),
\end{aligned}
$$

we have $\triangle_{21} \rightarrow 0$. Next we consider the term $\triangle_{22}$. From (2.6), we have

$$
\triangle_{22}=\frac{\lambda^{2}}{2}\left|\frac{1}{n} \sum_{j=1}^{n} \mathbb{E} Y_{j}^{2}-\sigma^{2}(x)\right| \rightarrow 0 .
$$

For the term $\triangle_{1}$, by the elementary inequality

$$
\left|e^{x}-1-x\right| \leqslant \frac{x^{2}}{2} e^{|x|} \quad \text { for all } x \in \mathbb{R},
$$

and (2.4), for all $n$ large enough we have

$$
\sup _{1 \leqslant j \leqslant n}\left|\mathbb{E} \exp \left(\lambda \frac{b_{n}}{\sqrt{n}} Y_{j}\right)-1\right| \leqslant C_{2} \frac{\lambda^{2} b_{n}^{2}}{2 n} \sup _{1 \leqslant j \leqslant n} \mathbb{E} Y_{j}^{2} \leqslant C_{3} \frac{\lambda^{2} b_{n}^{2}}{n h_{n}} \rightarrow 0
$$

where $C_{2}$ and $C_{3}$ are positive constants depending on $K(\cdot)$. Therefore for all $n$ large enough,

$$
\sup _{1 \leqslant j \leqslant n}\left|\mathbb{E} \exp \left(\lambda \frac{b_{n}}{\sqrt{n}} Y_{j}\right)-1\right| \leqslant \frac{1}{2}
$$

Using the elementary inequality

$$
|\log (1+x)-x| \leqslant 2 x^{2} \quad \text { for }|x| \leqslant 1 / 2,
$$

and the conditions (2.7) and (2.8), for any $n$ large enough we have

$$
\begin{aligned}
\triangle_{1} & =\frac{1}{b_{n}^{2}} \sum_{j=1}^{n}\left|\log \mathbb{E} \exp \left(\frac{\lambda b_{n}}{\sqrt{n}} Y_{j}\right)-\left[\mathbb{E} \exp \left(\frac{\lambda b_{n}}{\sqrt{n}} Y_{j}\right)-1\right]\right| \\
& \leqslant \frac{2}{b_{n}^{2}} \sum_{j=1}^{n}\left(\mathbb{E} \exp \left(\frac{\lambda b_{n}}{\sqrt{n}} Y_{j}\right)-1\right)^{2} \\
& \leqslant C_{4} \frac{1}{b_{n}^{2}} \sum_{j=1}^{n} \frac{\lambda^{4} b_{n}^{4}}{n^{2}}\left(\mathbb{E} Y_{j}^{2}\right)^{2} \leqslant C_{5} \frac{\lambda^{4} b_{n}^{2}}{n h_{n}} \frac{1}{n} \sum_{j=1}^{n} \mathbb{E} Y_{j}^{2}
\end{aligned}
$$

where $C_{4}$ and $C_{5}$ are positive constants depending on $K(\cdot)$. By (2.4) and (2.6), we have $\triangle_{1} \rightarrow 0$. From the above discussion, we obtain the desired result 2.5. 
COROLlaRY 2.1. Under the assumptions in Theorems 2.1 and 2.2 assume further that

$$
\frac{\sqrt{n} h_{n}^{1 / 2+\beta}}{b_{n}} \rightarrow 0
$$

Then for any $r>0$, we have

$$
\lim _{n \rightarrow \infty} \frac{1}{b_{n}^{2}} \log \mathbb{P}\left(\frac{\sqrt{n h_{n}}}{b_{n}}\left|\hat{f}_{n}(x)-\gamma_{n} f(x)\right| \geqslant r\right)=-\frac{r^{2}}{2 \sigma^{2}(x)}
$$

where $\gamma_{n}=n^{-1} h_{n}^{-1 / 2} \sum_{j=1}^{n} h_{j}^{1 / 2}$.

Proof. From Theorems 2.1 and 2.2, it is enough to show

$$
\frac{\sqrt{n h_{n}}}{b_{n}}\left|\mathbb{E} \hat{f}_{n}(x)-\gamma_{n} f(x)\right| \rightarrow 0
$$

but the condition 2.9 guarantees this. The recursive estimator $\hat{f}_{n}(x)$ is not an (asymptotically) unbiased estimator of $f(x)$, but $\gamma_{n}^{-1} \hat{f}_{n}(x)$ is asymptotically unbiased. Hence we have the following corollary.

COROLlaRY 2.2. Under the assumptions in Theorems 2.1 and 2.2. set $h_{n}=$ $n^{-\gamma}$, where $0<\gamma<1$. Furthermore, for any $\beta>0$ such that $\gamma(\beta+1 / 2)<1 / 2$, take $b_{n}$ satisfying $b_{n}^{-1}=o\left(n^{\gamma(\beta+1 / 2)-1 / 2}\right)$ and $b_{n}=o\left(n^{(1-\gamma) / 2}\right)$. Then for any $r>0$, we have

$$
\lim _{n \rightarrow \infty} \frac{1}{b_{n}^{2}} \log \mathbb{P}\left(\frac{\sqrt{n^{1-\gamma}}}{b_{n}}\left|\gamma_{n}^{-1} \hat{f}_{n}(x)-f(x)\right| \geqslant r\right)=-\frac{r^{2}(1-\gamma / 2)^{2}}{2 \sigma^{2}(x)}
$$

where $\gamma_{n}=n^{-1} h_{n}^{-1 / 2} \sum_{j=1}^{n} h_{j}^{1 / 2}$.

Proof. It is easy to see that the conditions 2.3, 2.4 and (2.9) hold and

$$
\gamma_{n} \rightarrow \frac{1}{1-\gamma / 2}
$$

So from Corollary 2.1, we get the desired result.

REMARK 2.2. Assume that for some $l>0$,

$$
\frac{1}{n} \sum_{j=1}^{n}\left(h_{j} / h_{n}\right)^{l} \rightarrow \beta_{l} \quad \text { as } n \rightarrow \infty .
$$

Masry [10] pointed out that under some conditions the recursive estimator $\hat{f}_{n}(x)$ is not an asymptotically unbiased estimator of $f(x)$. However, it is clear that after a simple scaling,

$$
\hat{\hat{f}}_{n}(x)=\frac{\hat{f}_{n}(x)}{\beta_{1 / 2}},
$$


$\hat{\hat{f}}_{n}(x)$ is an asymptotically unbiased estimator of $f(x)$. When $K(\cdot)$ is even symmetric, the dominant terms of the bias of $\tilde{f}_{n}(x)$ and $\hat{\hat{f}}_{n}(x)$ are

$$
\begin{aligned}
& \operatorname{bias}\left[\hat{\hat{f}}_{n}(x)\right] \sim \frac{c_{2} \beta_{2.5} f^{(2)}(x)}{2 \beta_{0.5}} h_{n}^{2}, \\
& \operatorname{bias}\left[\tilde{f}_{n}(x)\right] \sim \frac{c_{2} \beta_{2} f^{(2)}(x)}{2} h_{n}^{2} .
\end{aligned}
$$

If we take $h_{n}=n^{-\nu}$, then $\tilde{f}_{n}(x)$ will generally have smaller bias than that of $\hat{\hat{f}}_{n}(x)$. Furthermore, Masry [10] showed that $\tilde{f}_{n}(x)$ has a larger variance than $\hat{\hat{f}}_{n}(x)$ under the strong mixing condition for the samples $\left\{X_{i}, i \geqslant 1\right\}$.

\section{LARGE DEVIATION PRINCIPLE}

In this section, we assume that $K$ is a Borel function and the following conditions are satisfied:

(L1) The density function $f$ is bounded and $K(\cdot)$ is a non-negative function such that for any $t \geqslant 0$,

$$
\int_{\mathbb{R}}\left(e^{t K(z)}-1\right) d z<\infty
$$

$\left(\mathbf{L 1}^{\prime}\right) K(\cdot)$ is a non-negative function such that for any $t \geqslant 0$,

$$
\int_{\mathbb{R}}\left(e^{t K(z)}-1\right) d z<\infty, \quad \lim _{|z| \rightarrow \infty}|z|\left(e^{t K(z)}-1\right)=0 .
$$

(L2) $h_{n}=n^{-\alpha}$ with $0<\alpha<1$.

To state the large deviation principle for the estimator $\hat{f}_{n}$, we need the following lemmas. Their proofs are elementary, but we give them for completeness.

LEMMA 3.1. Let $a_{n}$ be a sequence of positive numbers such that $a_{n} \rightarrow 0$. If (L1) or (L1') holds, then for each continuity point $x$ of $f(\cdot)$ and for any $t>0$, we have

$$
\begin{aligned}
\lim _{n \rightarrow \infty} \int_{\mathbb{R}}\left(e^{t K(z)}-1\right) f\left(x-a_{n} z\right) d z & =f(x) \int_{\mathbb{R}}\left(e^{t K(z)}-1\right) d z, \\
\lim _{n \rightarrow \infty} \int_{\mathbb{R}} K(z) f\left(x-a_{n} z\right) d z & =f(x) \int_{\mathbb{R}} K(z) d z .
\end{aligned}
$$

Proof. (1) Assume that (L1) is satisfied. By the dominated convergence theorem, the claim (3.2) holds. Furthermore, by using the inequality $1+x \leqslant e^{x}$ for all $x \in \mathbb{R}$, we have

$$
\int_{\mathbb{R}} K(z) d z \leqslant \int_{\mathbb{R}}\left(e^{K(z)}-1\right) d z<\infty
$$


which implies (3.3) by the dominated convergence theorem.

(2) Assume that the condition $\left(\mathbf{L} \mathbf{1}^{\prime}\right)$ is satisfied. For any $\delta>0$, we have

$$
\begin{aligned}
\left|\int_{\mathbb{R}}\left(e^{t K(z)}-1\right)\left(f\left(x-a_{n} z\right)-f(x)\right) d z\right| & \\
\leqslant & \int_{\left|a_{n} z\right| \leqslant \delta}\left(e^{t K(z)}-1\right)\left|f\left(x-a_{n} z\right)-f(x)\right| d z \\
& +\int_{\left|a_{n} z\right|>\delta}\left(e^{t K(z)}-1\right)\left|f\left(x-a_{n} z\right)-f(x)\right| d z \\
\leqslant & \sup _{\left|a_{n} z\right| \leqslant \delta}\left|f\left(x-a_{n} z\right)-f(x)\right| \int_{\left|a_{n} z\right| \leqslant \delta}\left(e^{t K(z)}-1\right) d z \\
& +\int_{\left|a_{n} z\right|>\delta} z\left(e^{t K(z)}-1\right) \frac{f\left(x-a_{n} z\right)}{z} d z+f(x) \int_{\left|a_{n} z\right|>\delta}\left(e^{t K(z)}-1\right) d z \\
\leqslant & \sup _{|y| \leqslant \delta}|f(x-y)-f(x)| \int\left(e^{t K(z)}-1\right) d z \quad \int_{\mathbb{R}}\left(e^{t K(z)}-1\right) d z, \\
& +\frac{1}{\delta} \sup _{\left|a_{n} z\right|>\delta}\left[|z|\left(e^{t K(z)}-1\right)\right] \int_{|z-x|>\delta} f(z) d z+f(x)
\end{aligned}
$$

which tends to 0 if one lets first $n \rightarrow \infty$, and then $\delta \rightarrow 0$. From the inequality $1+x \leqslant e^{x}$ for all $x \in \mathbb{R}$, the limit (3.3) holds by a similar proof.

LEMMA 3.2. Suppose that either (L1) holds, or $K(\cdot)$ is a bounded Borel function. Then for each $x \in \mathbb{R}$ and for any $t, m>0$, we have

$$
\mathbb{E} e^{t K\left(x-m X_{1}\right)}<\infty .
$$

Proof. (1) Assume that (L1) is satisfied. Then

$$
\mathbb{E}\left(e^{t K\left(x-m X_{1}\right)}-1\right)=\frac{1}{m} \int_{\mathbb{R}}\left(e^{t K(u)}-1\right) f\left(\frac{x-u}{m}\right) d u,
$$

which yields the claim (3.4) by the boundedness of $f$ and condition (3.1).

(2) Assume that $K(\cdot)$ is a bounded Borel function. Then

$$
\int_{\mathbb{R}}\left(e^{t K(z)}-1\right) f(x-m z) d z=\frac{1}{m} \int_{\mathbb{R}}\left(e^{t K\left(\frac{x-u}{m}\right)}-1\right) f(u) d u \leqslant \frac{C}{m}
$$

where $C$ is a positive constant depending on $K$.

THEOREM 3.1. Suppose that either (L1)-(L2) or (L1')-(L2) hold and $K(\cdot)$ is a bounded Borel function. Then for each continuity point $x$ of $f(\cdot)$ and for any $r>0$, we have

$$
\lim _{n \rightarrow \infty} \frac{1}{n^{1-\alpha}} \log \mathbb{P}\left(\left|\hat{f}_{n}(x)-\mathbb{E} \hat{f}_{n}(x)\right| \geqslant r\right)=-J(r, x)
$$


where

$$
J(r, x)=\sup _{\lambda \in \mathbb{R}}\left\{r \lambda-f(x) \int_{\mathbb{R}}\left[\int_{0}^{1} s^{-\alpha}\left(\exp \left(\lambda s^{\alpha / 2} K(z)\right)-1-\lambda s^{\alpha / 2} K(z)\right) d s\right] d z\right\} .
$$

Proof. We use the Gärtner-Ellis theorem [2, Theorem 2.3.6]. For any $j$, let

$$
K_{j}=K\left(\frac{x-X_{j}}{j^{-\alpha}}\right) .
$$

Then we have (here $h_{n}=n^{-\alpha}$ )

$$
\hat{f}_{n}(x)-\mathbb{E} \hat{f}_{n}(x)=\frac{1}{n^{1-\alpha / 2}} \sum_{j=1}^{n} j^{\alpha / 2}\left(K_{j}-\mathbb{E} K_{j}\right) .
$$

Now we need to compute the logarithmic moment generating function of $\hat{f}_{n}(x)-\mathbb{E} \hat{f}_{n}(x)$. For any $\lambda \in \mathbb{R}$, we have

$$
\begin{aligned}
\Lambda_{n}(\lambda):= & \frac{1}{n^{1-\alpha}} \log \mathbb{E} \exp \left(\frac{\lambda}{n^{\alpha / 2}} \sum_{j=1}^{n} j^{\alpha / 2}\left(K_{j}-\mathbb{E} K_{j}\right)\right) \\
= & \frac{1}{n^{1-\alpha}} \sum_{j=1}^{n}\left[\log \mathbb{E} \exp \left(\frac{\lambda j^{\alpha / 2}}{n^{\alpha / 2}} K_{j}\right)-\left(\mathbb{E} \exp \left(\frac{\lambda j^{\alpha / 2}}{n^{\alpha / 2}} K_{j}\right)-1\right)\right] \\
& +\frac{1}{n^{1-\alpha}} \sum_{j=1}^{n}\left[\left(\mathbb{E} \exp \left(\frac{\lambda j^{\alpha / 2}}{n^{\alpha / 2}} K_{j}\right)-1\right)-\frac{f(x)}{j^{\alpha}} \int_{\mathbb{R}}\left(\exp \left(\frac{\lambda j^{\alpha / 2}}{n^{\alpha / 2}} K(z)\right)-1\right) d z\right] \\
& +\frac{1}{n^{1-\alpha}} \sum_{j=1}^{n} \frac{f(x)}{j^{\alpha}} \int_{\mathbb{R}}\left(\exp \left(\frac{\lambda j^{\alpha / 2}}{n^{\alpha / 2}} K(z)\right)-1-\frac{\lambda j^{\alpha / 2}}{n^{\alpha / 2}} K(z)\right) d z \\
& +\frac{1}{n^{1-\alpha}} \sum_{j=1}^{n} \frac{\lambda j^{\alpha / 2}}{n^{\alpha / 2}}\left(\frac{f(x)}{j^{\alpha}} \int_{\mathbb{R}} K(z) d z-\mathbb{E} K_{j}\right) \\
= & I_{1}+I_{2}+I_{3}+I_{4} .
\end{aligned}
$$

By Lemma 3.1, for any $\varepsilon>0$ there exists a positive constant $N_{0}$ such that for $N_{0} \leqslant j \leqslant n$ we have

$$
\begin{aligned}
& \quad\left|\mathbb{E} \exp \left(\frac{\lambda j^{\alpha / 2}}{n^{\alpha / 2}} K_{j}\right)-1\right| \\
& =j^{-\alpha}\left|\int_{\mathbb{R}}\left(\exp \left(\frac{\lambda j^{\alpha / 2}}{n^{\alpha / 2}} K(z)\right)-1\right) f\left(x-j^{-\alpha} z\right) d z\right| \\
& \leqslant j^{-\alpha}\left|\int_{\mathbb{R}}\left(e^{|\lambda| K(z)}-1\right) f\left(x-j^{-\alpha} z\right) d z\right| \leqslant j^{-\alpha}\left(\varepsilon+f(x) \int_{\mathbb{R}}\left(e^{|\lambda| K(z)}-1\right) d z\right) .
\end{aligned}
$$


Now we can choose $N_{1}$ such that

$$
\left|\mathbb{E} \exp \left(\frac{\lambda j^{\alpha / 2}}{n^{\alpha / 2}} K_{j}\right)-1\right| \leqslant 1 / 2 \quad \text { for } N_{1} \leqslant j \leqslant n .
$$

By using the elementary inequality

$$
|\log (1+x)-x| \leqslant 2 x^{2} \quad \text { for }|x| \leqslant 1 / 2,
$$

for $N:=\max \left\{N_{0}, N_{1}\right\} \leqslant j \leqslant n$ we have

$$
\begin{aligned}
& \left|\log \mathbb{E} \exp \left(\frac{\lambda j^{\alpha / 2}}{n^{\alpha / 2}} K_{j}\right)-\left(\mathbb{E} \exp \left(\frac{\lambda j^{\alpha / 2}}{n^{\alpha / 2}} K_{j}\right)-1\right)\right| \\
& \quad \leqslant 2\left(\mathbb{E} \exp \left(\frac{\lambda j^{\alpha / 2}}{n^{\alpha / 2}} K_{j}\right)-1\right)^{2} \leqslant 2 j^{-2 \alpha}\left(\varepsilon+f(x) \int_{\mathbb{R}}\left(e^{|\lambda| K(z)}-1\right) d z\right)^{2} .
\end{aligned}
$$

Furthermore, by Lemma 3.2, there exists a positive constant $C$ depending on $K, N, \lambda$ and $x$ such that

$$
\begin{aligned}
I_{1}= & \frac{1}{n^{1-\alpha}} \sum_{j=1}^{n}\left[\log \mathbb{E} \exp \left(\frac{\lambda j^{\alpha / 2}}{n^{\alpha / 2}} K_{j}\right)-\left(\mathbb{E} \exp \left(\frac{\lambda j^{\alpha / 2}}{n^{\alpha / 2}} K_{j}\right)-1\right)\right] \\
\leqslant & \frac{1}{n^{1-\alpha}} \sum_{j=1}^{N}\left[\log \mathbb{E} \exp \left(\frac{\lambda j^{\alpha / 2}}{n^{\alpha / 2}} K_{j}\right)-\left(\mathbb{E} \exp \left(\frac{\lambda j^{\alpha / 2}}{n^{\alpha / 2}} K_{j}\right)-1\right)\right] \\
& +\frac{1}{n^{1-\alpha}} \sum_{j=N+1}^{n}\left[\log \mathbb{E} \exp \left(\frac{\lambda j^{\alpha / 2}}{n^{\alpha / 2}} K_{j}\right)-\left(\mathbb{E} \exp \left(\frac{\lambda j^{\alpha / 2}}{n^{\alpha / 2}} K_{j}\right)-1\right)\right] \\
\leqslant & \frac{C N}{n^{1-\alpha}}+\frac{2}{n^{1-\alpha}} \sum_{j=N+1}^{n} j^{-2 \alpha}\left(\varepsilon+f(x) \int_{\mathbb{R}}\left(e^{|\lambda| K(z)}-1\right) d z\right)^{2},
\end{aligned}
$$

which yields $I_{1} \rightarrow 0$. For the terms $I_{2}$ and $I_{4}$, arguing similarly we have

$$
I_{2}=\frac{1}{n^{1-\alpha}} \sum_{j=1}^{n} j^{-\alpha}\left[\int_{\mathbb{R}}\left(\exp \left(\frac{\lambda j^{\alpha / 2}}{n^{\alpha / 2}} K(z)\right)-1\right)\left(f\left(x-j^{-\alpha} z\right)-f(x)\right) d z\right] \rightarrow 0
$$

and

$$
I_{4}=\frac{\lambda}{n^{1-\alpha / 2}} \sum_{j=1}^{n} j^{-\alpha / 2}\left(f(x) \int_{\mathbb{R}} K(z) d z-\int_{\mathbb{R}} K(z) f\left(x-j^{-\alpha} z\right) d z\right) \rightarrow 0 .
$$

For the term $I_{3}$, by the elementary inequality

$$
\left|e^{x}-1-x\right| \leqslant \frac{x^{2}}{2} e^{|x|} \quad \text { for all } x \in \mathbb{R}
$$


we have

$$
\frac{1}{n} \sum_{j=1}^{n}\left(\frac{n}{j}\right)^{\alpha}\left|\exp \left(\lambda\left(\frac{j}{n}\right)^{\alpha / 2} K(z)\right)-1-\lambda\left(\frac{j}{n}\right)^{\alpha / 2} K(z)\right| \leqslant \frac{\lambda^{2}}{2} K^{2}(z)
$$

Since (L1) or (L1') implies $\int_{\mathbb{R}} K^{2}(z) d z<\infty$, from some analytic considerations and the dominated convergence theorem we have

$$
\begin{aligned}
I_{3} & =\frac{1}{n} \sum_{j=1}^{n}\left(\frac{n}{j}\right)^{\alpha} \int_{\mathbb{R}}\left(\exp \left(\lambda\left(\frac{j}{n}\right)^{\alpha / 2} K(z)\right)-1-\lambda\left(\frac{j}{n}\right)^{\alpha / 2} K(z)\right) f(x) d z \\
& \rightarrow f(x) \int_{\mathbb{R}}\left[\int_{0}^{1} s^{-\alpha}\left(\exp \left(\lambda s^{\alpha / 2} K(z)\right)-1-\lambda s^{\alpha / 2} K(z)\right) d s\right] d z .
\end{aligned}
$$

From the above discussion, the desired result follows.

COROLLARY 3.1. Under the conditions (L1')-(L2), assume that $K$ is bounded and has Fourier transform $K^{*}(u)=\int_{-\infty}^{\infty} e^{-i u y} K(y) d y$. Suppose further that for some positive constant $\beta$,

$$
\lim _{u \rightarrow 0}\left[1-K^{*}(u)\right] /|u|^{\beta}=k_{\beta}
$$

is finite and

$$
f^{(\beta)}(x)=-\frac{1}{2 \pi} \int_{-\infty}^{\infty} e^{-i u x}|u|^{\beta} \phi(u) d u
$$

exists, where $\phi(\cdot)$ denotes the characteristic function of the random variable $X$ with density function $f(x)$. Then for any $\alpha$ with $\alpha\left(\beta+2^{-1}\right)<1$ and any $r>0$, we have

$$
\lim _{n \rightarrow \infty} \frac{1}{n^{1-\alpha}} \log \mathbb{P}\left(\left|\hat{f}_{n}(x)-(1-\alpha / 2)^{-1} f(x)\right| \geqslant r\right)=-J(r, x)
$$

where $J(r, x)$ is defined in Theorem 3.1

Proof. Note that the condition (L1') implies

$$
\int_{\mathbb{R}} K(z) d z<\infty \text { and } \lim _{|z| \rightarrow \infty}|z| K(z)=0
$$

Then the corollary can be deduced from Theorems 2.1 and 3.1

Acknowledgments. The authors are grateful to the referees for their valuable reports which improved the presentation of this work. 


\section{REFERENCES}

[1] M. Csörgö and L. Horváth, Central limit theorems for $L_{p}$-norms of density estimators, Probab. Theory Related Fields 80 (1988), 269-291.

[2] A. Dembo and O. Zeitouni, Large Deviations Techniques and Applications, Springer, Berlin, 2010.

[3] F. Q. Gao, Moderate deviations and large deviations for kernel density estimators, J. Theoret. Probab. 16 (2003), 401-418.

[4] F. Q. Gao, Moderate deviations and law of the iterated logarithm in $L_{1}\left(\mathbb{R}^{d}\right)$ for kernel density estimators, Stochastic Process. Appl. 118 (2008), 452-473.

[5] H. Y. Liang and J. Baek, Asymptotic normality of recursive density estimates under some dependence assumptions, Metrika 60 (2004), 155-166.

[6] H. Y. Liang and J. Baek, Berry-Esseen bounds for density estimates under NA assumption, Metrika 68 (2008), 305-322.

[7] D. Louani, Large deviations limit theorems for the kernel density estimator, Scand. J. Statist. 25 (1998), 243-253.

[8] D. Louani, Large deviations for the $L_{1}$-distance in kernel density estimation, J. Statist. Plann. Inference 90 (2000), 177-182.

[9] Z. D. Lu, Asymptotic normality of kernel density estimators under dependence, Ann. Inst. Statist. Math. 53 (2001), 447-468.

[10] E. Masry, Recursive probability density estimation for weakly dependent stationary processes, IEEE Trans. Inform. Theory 32 (1986), 254-267.

[11] E. Masry and L. Györfi, Strong consistency and rates for recursive probability density estimators of stationary processes, J. Multivariate Anal. 22 (1987), 79-93.

[12] A. Mokkadem, M. Pelletier and B. Thiam, Large and moderate deviations principles for recursive kernel estimator of a multivariate density and its partial derivatives, Serdica Math. J. 32 (2006), 323-354.

[13] A. Mokkadem, M. Pelletier and J. Worms, Large and moderate deviations principles for kernel estimation of a multivariate density and its partial derivatives, Austral. New Zealand J. Statist. 47 (2005), 489-502.

[14] E. Parzen, On estimation of a probability density function and mode, Ann. Math. Statist. 33 (1962), 1065-1076.

[15] M. Rosenblatt, Remarks on some nonparametric estimates of a density function, Ann. Math. Statist. 27 (1956), 832-837.

[16] E. J. Wegman and H. I. Davies, Remarks on some recursive estimators of a probability density, Ann. Statist. 7 (1979), 316-327.

[17] C. T. Wolverton and T. J. Wagner, Asymptotically optimal discriminant functions for pattern classification, IEEE Trans. Information Theory 15 (1969), 258-265.

[18] H. Yamato, Sequential estimation of a continuous probability density function and mode, Bull. Math. Statist. 14 (1971), 1-12.

[19] D. X. Zhang and H. Y. Liang, Recursive density estimation of NA samples, Chinese J. Appl. Probab. Statist. 24 (2008), 123-134.

Yu Miao, Qinghui Gao, Jianyong Mu, Conghui Deng

College of Mathematics and Information Science

Henan Normal University

Xinxiang, Henan Province, 453007, China

E-mail: yumiao728@gmail.com, qinghuigaocb@163.com, jianyongmu@163.com, conghuideng820@163.com

Received 28.2.2018; revised version 7.10 .2018 
